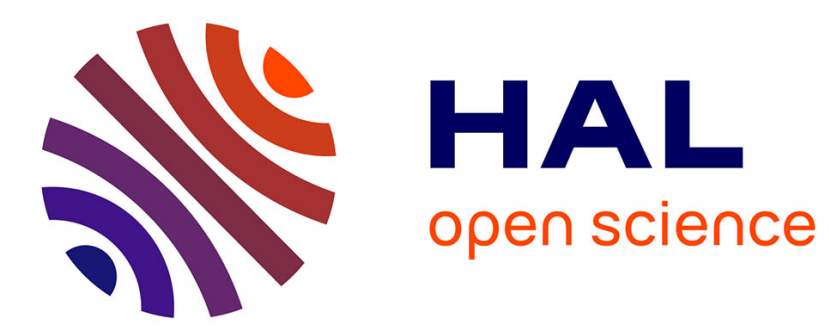

\title{
Dispositif multiangulaire pour les mesures d'asymétrie en neutrons
}

\author{
R. Sené, J. Kahane, P. Delpierre, M. Heyman
}

\section{To cite this version:}

R. Sené, J. Kahane, P. Delpierre, M. Heyman. Dispositif multiangulaire pour les mesures d'asymétrie en neutrons. Revue de Physique Appliquée, 1969, 4 (2), pp.245-246. 10.1051/rphysap:0196900402024500 . jpa-00243243

\section{HAL Id: jpa-00243243 https://hal.science/jpa-00243243}

Submitted on 1 Jan 1969

HAL is a multi-disciplinary open access archive for the deposit and dissemination of scientific research documents, whether they are published or not. The documents may come from teaching and research institutions in France or abroad, or from public or private research centers.
L'archive ouverte pluridisciplinaire HAL, est destinée au dépôt et à la diffusion de documents scientifiques de niveau recherche, publiés ou non, émanant des établissements d'enseignement et de recherche français ou étrangers, des laboratoires publics ou privés. 


\title{
DISPOSITIF MULTIANGULAIRE POUR LES MESURES D'ASYMÉTRIE EN NEUTRONS
}

\author{
R. SENÉ, J. KAHANE, P. DELPIERRE et M. HEYMAN, \\ Physique Atomique et Moléculaire, Collège de France.
}

\begin{abstract}
Résumé. - Le dispositif d'analyse de temps-de-vol multiangulaire décrit permet de travailler avec 8 voies de détection et un sélecteur 400 canaux. La résolution du temps-de-vol cumulé sur un mois s'élargit de 1,5 ns (résolution instantanée) à 2,5 ns.
\end{abstract}

Abstract. - The multiangular time-of-flight device described allows one to work with eight detection lines and a 400 channels pulse-height analyzer. The flight-time resolution throughout one month increases from $1.5 \mathrm{~ns}$ (instantaneous resolution) to $2.5 \mathrm{~ns}$.

Pour mesurer l'asymétrie dans la distribution angulaire des neutrons polarisés de $14 \mathrm{MeV}$ diffusés élastiquement et inélastiquement, nous avons mis en place un dispositif de spectrométrie multiangulaire par tempsde-vol. Les caractéristiques du générateur de neutrons polarisés nous imposent de cumuler sur une centaine d'heures, réparties sur plusieurs semaines, des mesures élémentaires enregistrées dans des conditions aussi voisines que possible. Nous avons donc multiplié au maximum le nombre de détecteurs. Nous travaillons en géométrie ouverte avec des barres d'ombre. Pour chaque mesure, l'implantation géométrique des détecteurs et des barres d'ombre (choix des angles et forme des barres) est étudiée sur maquette.

I. Détecteurs. - Chaque détecteur est constitué d'un bloc de NE 102 .A de $100 \times 100 \times 50 \mathrm{~cm}^{3}$ ou $100 \times 50 \times 50 \mathrm{~cm}^{3}$, associé à un photomultiplicateur 56 AVP.03. Les dimensions ont été choisies comme étant un compromis entre le rendement de la détection, la précision angulaire et la résolution du tempsde-vol.

II. Traitement d'une voie [1]. - Nous prélevons, à l'anode de chaque PM, deux impulsions identiques. L'une, après une double différenciation passive (par ligne à retard), attaque un circuit de mise en forme à diode tunnel à seuil très bas et fournit l'information en temps $(2 \mathrm{~V}, 10 \mathrm{~ns})$. Le déclenchement est réglé au voisinage du point d'inflexion du front de montée de l'impulsion d'origine, de façon à minimiser les fluctuations en temps dues à la dynamique d'entrée $(1 / 20)$. La seconde impulsion sert à fixer le seuil en énergie. Après une légère intégration $(R C=30 \mathrm{~ns})$ permet- tant d'améliorer la définition du seuil (sensibilité $\pm 10 \mathrm{keV}$ électron) tout en conservant une rapidité suffisante (occupance $100 \mathrm{~ns}$ ), le signal attaque un discriminateur à diode tunnel. Le signal de sortie autorise la transmission du signal emportant l'information en temps. Un troisième signal prélevé sur la douzième dynode est prévu pour l'identification de la particule détectée (n ou $\gamma$ ) [2].

III. Temps-de-vol une voie. - La mesure du tempsde-vol utilise la méthode classique de la particule associée. La conversion temps-amplitude est démarrée par le signal de la voie $\alpha$ et est stoppée par le signal d'une des voies neutrons. Le convertisseur tempsamplitude (GTA) est du type bistable. Jusqu'à un taux de 1000 événements par seconde, on a une plage

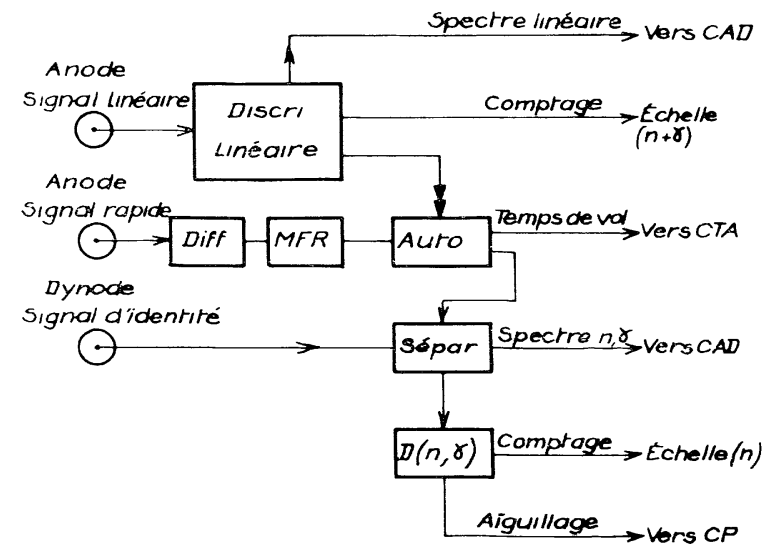

FIG. 1. - Logique une roie "neutrons". 


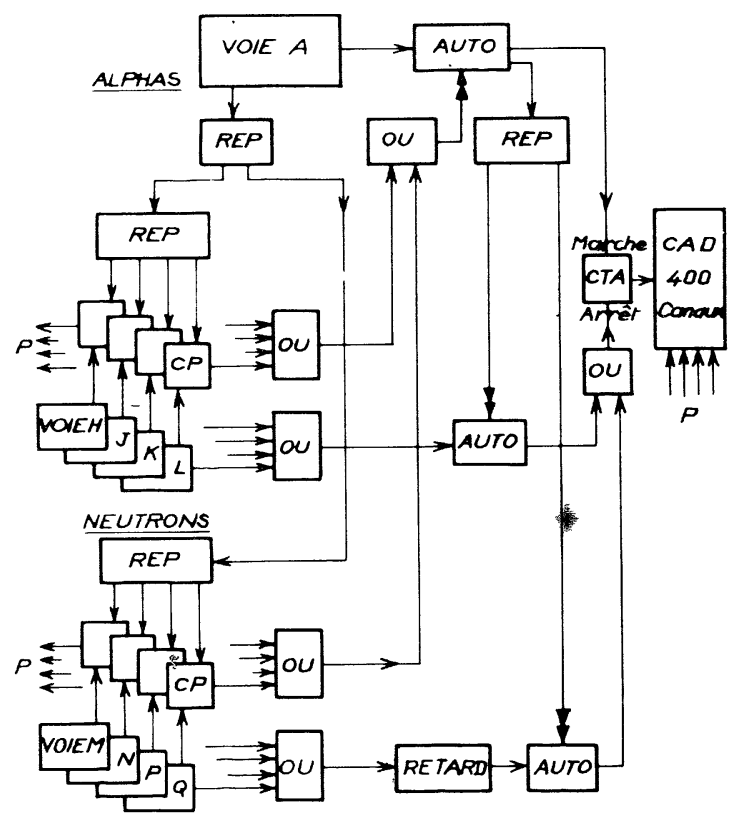

FIG. 2. - Logique dutemps de vol à 8 détecteurs. de linéarité de 200 ns définie à mieux que $1 \%$. Afin de ne pas saturer le convertisseur, le signal de marche n'est transmis que si un signal arrêt est présent. Les impulsions de sorties sont analysées par un sélecteur 400 canaux TMC. Les signaux de coïncidence $\alpha-n$ (CP) servent d'aiguillage pour les quatre aires de stockage de 100 canaux.

IV. Dispositif multiangulaire. - Nous avons travaillé de cette façon avec quatre détecteurs. L'enregistrement de deux spectres par plage de 100 canaux nous a permis de doubler le nombre de détecteurs. En effet, dans nos conditions expérimentales, une plage de 50 canaux correspondant à 50 ns pouvait suffire. Pour éviter le chevauchement des spectres, une ouverture de porte de durée donnée déclenchée par le signal marche filtre les signaux de stop. Un simple ajustement des retards permet de régler la séparation à mi-plage. La résolution instantanée mesurée sur un spectre direct $\alpha-n$ varie de 1,4 à 1,8 ns suivant les voies. En diffusion, en raison des dispersions dans le diffuseur et des dérives, le pic élastique s'élargit jusqu'à environ 2,5 ns (spectres cumulés sur quatre semaines).

\section{BIBLIOGRAPHIE}

[1] Rapport interne P.A.M., Collège de France, 1968.

[2] KAHANE (J.) et al., ce colloque. 